\title{
Distant learning challenges and solutions: Incorporation of 3D protein visualisation in an undergraduate pharmacy medicinal chemistry course
}

Heba Abdel-Halim

Faculty of Pharmacy and Medical Sciences, University of Petra, Jordan

\section{Keywords}

Medicinal Chemistry

Distant Learning

Three-dimensional (3D) Visualisation

Team-based Learning

Jordan

Corresponding author:

habdelhalim@uop.edu.jo
Summary: The COVID-19 pandemic made distance learning an essential medium for teaching worldwide. The use of three-dimensional protein visualisation in teaching undergraduate medicinal chemistry courses was shown to be highly effective in increasing the students' understanding and interest in the topic. The course was designed to use the visualisation tools in lectures, and students would then use them to study drug-target interactions and present their work as a part of the course assessment. With the pandemic induced-lockdown imposed early in the term, the faculty were faced with many challenges to keep the use of tools as part of the course. The lack of direct contact, differences in students' computer literacy and availability of internet connections, in addition to the need for appropriate mediums for assignment presentations and assessment, were major concerns. In this case study the author presents the Faculty of Pharmacy and Medical Sciences at the University of Petra's approaches to overcome the challenges faced in the course delivery and assessment.

\section{Background and Context}

Binding of a drug onto its target is highly dependent, not only on the drug's chemistry, but also on its three-dimensional (3D) structure (Satyanarayanajois \& Hill, 2011). Students' understanding of the molecular aspects of drug-target interactions is one of the major challenges facing the teaching of medicinal chemistry (Tavares et al. 2017). The use of $3 \mathrm{D}$ protein visualisation tools in medicinal chemistry classes and in the assignments have helped students develop a better understanding of the molecular basis of the effect of the drug action; in addition it provided students with the tools to correlate the therapeutic outcomes to the drugs' chemistry (Abdel-Halim, 2020). The 3D visualisation was an essential tool in the delivery of the course; in lectures students were shown a 3D presentation of the studied drugs while bound to their site of action. This was used to establish an understanding of bonds important for the drugs' effect and hence students were able to conclude the structure activity relationship (SAR) based on the types of interactions formed. In addition, the 'macromolecular drug targets assignment' part of the course provided the students with practical experience in using new in silico techniques to study and understand the effect of the different drug-target interactions on drugs' activity and effect. The use of advanced software can be troublesome for students; many measures were therefore taken and were found efficient in combating the challenges faced after introducing the assignments (Abdel-Halim, 2020). The COVID-19 pandemic lockdown and the move to distance learning presented new challenges to introducing the new techniques; however new methods have ensured an effective learning process.

\section{Educational Description}

Many challenges to the teaching of the medicinal chemistry course and meeting the requirements of the assignments needed to be surmounted. In this case study the author presents University of Petra's Faculty of Pharmacy and Medical Sciences' actions to overcome the challenges on two topics caused by the COVID-19 lockdown: 1 ) the use of 3D 
visualisation tools to correlate drug-target interactions to drug activity and SAR; and 2) keeping the 'macromolecular drug targets assignment' as part of the course's pass requirements.

\section{Use of 3D visualisation tools}

A large number of academics around the world were unprepared for distance learning (DL) and were unfamiliar with the available online teaching tools. The medicinal chemistry course lectures continued in the form of live podcasts using the available $\mathrm{DL}$ tools. 3D protein visualisation software was still used in the lectures. This was made possible by using the 'Share Screen' option available in many of the online meeting interfaces. As the faculty proceeded in the course some students with weaker internet connections suffered from a time lag in the videos which prevented them from following the elaboration on the $3 D$ structures. Therefore, two-dimensional (2D) screenshots of the 3D structures, taken from different perspectives, were prepared and introduced to the lecture notes and hence students were able to follow explanations by following $2 \mathrm{D}$ and/or the structures.

\section{Macromolecular drug targets assignment}

This was the most challenging aspect of the DL process. In normal course situations, help would be given to students in different forms, i.e. providing students with comprehensive step-by-step manuals and training sessions on the use of the software and protein data bank or one-on-one help to resolve any problems they encountered in their work. DL presented many challenges, mainly the lack of direct contact; differences in computer literacy and availability of stable internet connections among students; and the need for a medium for assignments, results presentations and marking. The team-based learning (TBL) concept was found to be the most appropriate to bridge the gaps between students' skills. Students were asked to work in teams of five to six ensuring that each team had a diversity in computer skills, the availability of a stable internet connection and a computer. The TBL helped stimulate collaboration within each team, with students learning from each other and providing the extra help required for the assignment training. After forming teams, the assignment hand-out and a video recording detailing all the steps were made available to students. A number of live online meetings were set at different stages of the assignment timeline for students to address any issues they faced. Students presented their work in the form of a scientific poster. Individual online meetings were organised for each team and students shared their posters with each student presenting their assigned part as agreed amongst themselves. Students received frequent and immediate feedback during each session and their work was marked according to the pre-announced mark rubric. At the end of the semester, students evaluated the course and the new DL experience with a survey that included specific questions about the assignment and their experience with the 3D tools.

\section{Outcomes and Recommendations}

The results of the survey showed that most students were satisfied with the course layout and the use of 3D protein visualisation tools to explain the drug-target interactions and SAR. The 3D tools have improved students understanding of medicinal chemistry. Most students preferred the depictions in the 3D format over the 2D format, however students who faced internet connection issues agreed that the 2D illustrations were helpful in keeping up with the live lectures.

Some students faced difficulties in using the software, with some not having access to high-speed internet. For these students forming teams to reflect diversity in skill, and availability of resources was found helpful and relived the stress of the assignment. Almost all of the students found the assignment manual, training video and live meetings sufficient in providing them with the required guidance and support. Most students, however, preferred direct face-toface contact for presenting their work. Most of the groups believed that having their peers with them would have provided them with more comfort and confidence. However, some thought that the online presentation had alleviated the social pressure and that they performed better than they would have in a traditional poster session.

The faculty's experience of $\mathrm{DL}$, despite being challenging, was found to fulfil the learning objectives of the course. TBL was used for the first time and the faculty will continue to use this approach in the future. The department would decrease the direct supervision of students to promote more accountability as they become more responsible for their own learning. Students will be allowed more time to experiment and try to find their own answers before they ask for help. Teamwork has fostered collaboration with their team members, and this has led to an enhanced learning experience.

TBL can be used in the pharmacy curriculum to promote students' critical thinking, collaboration, and to help them take responsibility for their own learning, enabling them to become lifelong learners.

\section{References}

Abdel-Halim, H. (2020). The Effectiveness of Using ThreeDimensional Visualization Tools to Improve Students' Understanding of Medicinal Chemistry and Advanced Drug Design Concepts. International Journal of Learning, Teaching and Educational Research, 19(4), 170-188. https://doi.org/10.26803/ij|ter.19.4.11

Satyanarayanajois, S., \& Hill, R. (2011). Medicinal chemistry for 2020. Future Medicinal Chemistry, 3(14), 1765-1786. https://doi.org/ $\underline{10.4155 / \mathrm{fmc} .11 .135}$

Tavares, M.T., Primi, M.C., Silva, N.A.T.F., Carvalho, C.F., Cunha, M.R., \& Parise-Filho, R. (2017). Using an in Silico Approach To Teach 3D Pharmacodynamics of the Drug-Target Interaction Process Focusing on Selective COX2 Inhibition by Celecoxib. Journal of Chemical Education, 94(3), 380-387. https://doi.org/10.1021/acs.jchemed. $\underline{6 b 00288}$ 